Summary Talk - Experimental ${ }^{\text {t }}$

\section{P. Kienle}

Technical University of Munich "unich, Germany

and

Lawrence Berkeley Laboratory University of California Ber "ley, California 94720

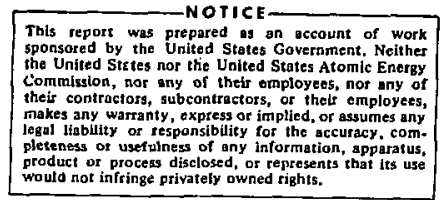

This report was prepared as an account of work 5ponsored by the United Stotes Government. Neither the United Stetes nor the United States Atomic Energy Commirston, nor any of thetr omployes, not sny of heis contractors, subcontractors, or thels employees, cgal linbllity or responsibility for the or assumes any pleteness of usefplness of any information, apparatus, would not infringe privately owned rights.

November 1972

I have the privilege to make the concluding remarks at this international conference on nuclear moments and nuclear structure. This conference seems to me more like an exhibition of fine arts, sponsored by an academy of oldest tradition and arranged by its granö master Professor Sugimoto from Osaka. On Monday the entrance hall was opened and it looked very spectacular to me. The lofty, glittering roof anchored to the $\mathrm{N}^{*}$-eternity was a kind gift to this exhibition by Gerry Brown. It was fimly suspended by that original architect from the Japanese pion Bauhaus, Professor Miyazawa. Then, the waster magician Yamazaki, to whom the exhibition was dedicated, displayed impressively his colorful kinetic meson art. Further on, the decorations were provided by the world's famous nuclear futurist, Lee Grodzins from the MIT, and finally the whole hall was filled by Mme. Wu's surrealistic muon dream. Those of you who know me, know that I like artists and their shows. They are my friends and they have my admiration for their sincere devotion in creating a new world full of miracles in which they live, often very far away from the newsmen, who are always around, the highways, the main streams and even their friends. That derotion I think is their strength but maybe their weakness at the same time.

Work performed under the auspices of the U. S. Atomic Energy Cormizsicn. 
Now let me tell you a little about what I have seen on ry rather casual walk through the halls with eyes of a very simple minded experimentalist, who still has the luck of being able to appreciate the excitement coming from developments based on very simple principles, devised to get answers conceruing very snall effects such as those caused by nuclesr moments. You know this is a field of very small effects, although it did not always sound that way when I was listening to my old historian friend Hans Frauenfelder. I still think that nuclear moments are rather unspectacular, but always very trustworthy, steady companions on the way of developing a better understanding of nuclei, particles, ators, molecules, and even condensed matter. I also believe that most of the beauty and excitement of tilis field is intimately connected with the methods which are very ingenious combinations of basic principles and phenomena of large variety and are also cleverly applied to other interesting branches of science such as biochemistry and solid state physics.

Let us look first at the oldest method of nuclear-moment research, the spectroscopy. You know it is still alive with the Mössbauer effect, ana muon and exotic atom spectroscopy. Even if Professor Matthias thinks that the Mössbauer effect is passé, there are still nice contributions to this conference on hyperfine anomalies of excited states and the shrinking of charge radii which became observable only because of an enormous improvement in the measuring precision. I mean, these effects are so small that one has to sharpen the old techniques to really get precise information. These very interesting results are difficult to get with any other technique and are complementary to the beautiful isomer shift work on $\gamma$-rays from muonic atoms. The analysis of these latter experiments, as presented by Professor Kankeleit, 
sheds light on the change of the second and mayte the third moment of the charge distribution which are caused by the nuclear rotation. I think more applications of the Mössbauer effect will almost certainly come, especially in connection with quadrupole moment ineasurements of isomers, which I shall discuss later. There may even be some very basic surprises in the air, such as that which came very recently, perfectly unexpected, from Potzel and Perlow of the Argonne National Laboratory. They did what $I$ think is a rather intricate but conventional measurement to find the sign of the quadrupole moment in $197_{\mathrm{Au}}$. They could determine that but, at the same time, they found that the Mössbauer lines in certain compounds were $20 \%$ narrower than expected from rather accurate lifetime measurements. Now regardless of the solution of this very strange phenomenon, which is extremely difficult to explain, I think such things bring new creative excitement with them, which is so badly nzoded in our stagnating scientific sxiety.

Let's go on to the muon spectroscopists. They came to us displaying very beautiful research on hyperfine anomalies of ground states and also excited states, which I think will reactivate an old field and provide a new dimension for magnetic-moment research by revealing information on magneticmoment distributions. Furthermore, we heard very exciting news for the efforts to measure the so far elusive ground-state quadrupole moments in a model independent way. The news came from the CERN group which succeeded in measuring and also especially in analyzing the qudrupole splittings of outer orbits in muonic atoms. There are certainly many open questions about their analysis and the extraction of unambiguous results. However, one has great hope that the

\footnotetext{
*W. Potzel and G. I. Perlow, Phys. Rev. Letters 29, 910 (1972).
} 
remaining problems can ve solved in time. If so, I think it woula have an enormous impact on quadrupole-moment research because i.t woulä allow one to extract field gradients, for example, in atoms and in solids. It would 1mprove the theoretical understanding of field gradients and it would, and this is most important, yield a basis for determining also quaarupole moments of excited states with the perturbed-angular-correlation method, i.e., if one knows the field gradients in a given environment, then one can extract the quadrupole monents of the excited states. In order to determine the quadrupole constants in various environments, which are then used for measuring quadrupole moments in excited states, one can use known techniques like quadrupole resonances, Mössbauer effect, nuclear alignment, and many others to get the final information. So this field is auite interesting because until now we really haven't had any means except Coulomb excitation to measure quadrupole moments in a model independent way.

In this context, I am tempted to drew your attention to a kind of spectroscopy which seems to be unknown even to specialists. The method has been sleeping very deeply in a two or three years old volume of Zeitschrift für Physik ${ }^{*}$; it was published by Heidemann, a student of Meier-Leibnitz. Let me explain his method to you, which I would like to call hyperfine neutron spectroscopy. The method is so beautiful that its main features deserve to be described. What does Heidemann do? He scatters slow neutrons from nuclei and in some of the scattering events the spins flip; i.e., both the nucleus and the neutron make spin transitions with $\Delta m=1$, but of opposite sign to conserve EnEuiar zomentum. Now assure that the m-state degeneracy of the nucleus is

\footnotetext{
A. lleidemann. Z. f. Physik 238, 208 (1970).
} 
removed in a solid by a hyperfine interaction, let's say, a quadrupole interaction. In scattering process involving a spin flip the energy is transierred between the neutron and the nucleus. So he used a highly monochromatic neutron beam produced by backscattering of very slow neutrons from a silicon crystal and he measured the energy of the scattered neutron by the same process. To measure the change in energy of the neutrons which were involved in a spin-flip scattering he uses a Doppler-shift technique just as in the Mössbauer effect. In his first experiment he achieved a resolution of some $10^{-7} \mathrm{eV}$ and he could indeed observe a hyperfine splitting. With the advent of the high flux reactors, I think this technique can be tremendously improved in intensity and also in resolution, so this may become a very powerful method for the measurement of quadrupole coupling constants.

Nov: I would like to proceed and look into the central hall of this exhibition, which displayed the numerous contributions of the polarization masters and the NMR artists. As you know, they use the most exotic combinations of all sorts of principles to measure g-factors and quadrupole-coupling constants of nuclear states over an enormous lifetime range, from stable oness down to those with lifetimes in the pico-second region. I am very sorry that I can only give a very inadequate presentation of their impressive achievements by restricting myself to some of the experimental developments, which seem for me to be the most characteristic ones of the field. The art of the measurements on unstable nuclear states is besed on combinations of simple principles like the production of alignment or polarization, its observation via the angular distribution of beta particles, gamme rays or other particles, and then the measurement of the change of the angular distribution procuced by interaction 
with electrommgnetic fields. Now let me brlefly review some of the newer developments of the experimental methods or developments which struck me must. The use of nuclear reactions to produce aligned or polarized nuclear states had an enormous effect on the variety of states which are now accessible for moment measurement. This puts us in a very fortunate situation in making choices according to our or even the theoreticians' taste in picking intertesting cases. Typical examples are Sugimoto's artistic measurements on the light isobars which shed light on basic problems of magnetic moments as well as on structural problems of the $p$ - and sd-shell nuclei. In context with these experiments, one may hope that the availability of high intersity poiarized neutron beams from the high flux reactors as well as polarized charge-particle beams from the many accelerators, which $\mathrm{n} \cdot$ have polarized scurces, may lead to substantial progress in measuring magnetic morents as well as quadrupole moments for short-lived activities by using essentially Sugimoto's techniques. Another striking example is the measurement of the g-facturs of highspin isomers around closed-shell nuclei, initiated by Yamazaki using the powerful tool of compound reactions to populate and align these states. I think this development led to the main theme of this conference, namely the question of the importance of mesonic exchange effects about which you heard such a lot and the problem of core polarization, on which theoreticians from this country like Miyazawa, Horie and Arima have worked a long time. Professor Yamazaki who, I think, is a real kinetic artist showed me a colorful figure which I would like to describe to you. He represented the well-known Schmidt lines by blue lines, then he added red lines which could be called Miyazawa lines because they contain the correction $\Delta \mathrm{g}_{\ell}=0.1$, which emerged from the 
experiments, which have been done to such a great extent recently. Now I think more progress, particularly in respect to the cholce of interesting cases, can be made if one uses the pulsed heavy-ion beams from $t$ e mary accelerators which now exist, like tandems, cyclotrons and linear accelergtors, and use these beams to populate rery specific states of interest with the best possible reaction one can find. In this way, it should be possible to get more information on neutron states and on both members of spin-orbit doublets near closed shell nuclei. Furthermore, it is quite clear that use of heavy-ion reactions and recoil methods will oper further possibilities of measuring moments of short-lived single particle states in heavy nuclei, around ${ }^{208} \mathrm{~Pb} s a y$, and of obtaining quite interesting information on all sorts of transition matrix elements.

Now I would shortly like to turn to another subject, namely the optical-pumping and atomic-beam methods combined with on-line mass-separators and eccelerators, which seem to provide a very important tool ror investigating nuclel far off the stability line whinh we really don't know very well. You may ask, why do you want to investigate such nuclei, I zean, when you don't even understand the ones along the stability line. However, I think Professor Otten's measurement on the very drastic increase of the nuclear charge radius between $187_{\mathrm{Hg} \text { and }} 185,183_{\mathrm{Hg}}$ is a very fine example of a result which was not really expected and which can be obtained by such methods.

Now let me turn to the actual measurement of quadrupole- and magneticinteraction constants with methods like spin-rotation, the stroboscopicrescrance technique, which was introduced by the Berlis group, and NMR. With these techniques one now has a very high degree of versatility and accuracy 
. Lnd they cover an enormous range of lifetimes and frequencles. Furthermore, the improved understanding of static and transient internal fields as well as the inte: ctions in highly ionized gtoms recoilins into gas or into vacuum hast frovided us with new tools for measurement of moments of highly excited states which are very short-livid. This will be especially true, I think, wher heavyion beams become more readily available. But already the results presented at this conference, for example, by Professor Hanna, are quite exciting anc. Indicate, I think, the beginning of a new area of nuclear moment research.

Finally, I should like to draw your attention to some duadrupole-moment measurements which nay indicate that a new area of research has already been started. It was shown first, I think, by a group in Stony Brook and then in Berlin and Munich that quadrupole interactions of isomers populated by compound reactions may be nicely studied with precession techniques. Now if field gradients can be determined from those developments which I menioried earlier, we have a way to measure the quisurupole moments of the high-spin states around closed-shell nuclei. When I prepared this talk, I didn't know if these measurements were as exciting as those which measure the corresponding g-factors, and I thought of suggesting that one should ask Arima or Gerry Brown about this. However, I wouldn't do that because I think I would measure them anyhow, but you have heard, I think, from Arima that these measurements may be quite interesting.

There are other examples which are concerned vith the question of coexistence of states with different leformations, such as the states in the actinides, for example, the fission isomers. It would certainly be interesting to learn more about then and to see if such states exist in other nuclei. There 
were recently two very nice measurements on states in ${ }^{117}$ In and ${ }^{115}$ In which gave very large quadrupole moments, although I don't remember the exact numbers. Along with the available spectroscopic information these experiments indicated the existence of a real deformed band. This may be a more general phenomenon in nuclei and 1 t could be investlgated with the methods which I have describea. Now we st1ll have some problems with fission isomers, and some of you may know that the moment of inertia of one of them, namely ${ }^{240} \mathrm{Pu}$, has been recently measured by specht and collaborators in Munich to be twice as big as the corresponding moment of inertia of the ground-state rotational band. Now it would be nice to learn more about these nuclei with these suppcsedly high moments of inertia $0 ;$ large deformations. In order to really pin down the whole phenomenon, one would like to investigate the odd nuclei. The states in these nuclei are usually populated with an $(\alpha, 2 n)$ or some kind of conpound reaction. The question is can we play the nuclear moment game and use all our spectroscopic tools to learn more about the spins, magnetic moments, and quadrupole moments of these states. Basically, these measurements can be done although I think they are very difficult - by measuring as a function of time the anisotropy of the fission fragments from these isomers which have been naturally aligned by the compound reactions. I don't knows if this would be very interesting but I think it would be possible to really pin down what is going on in these nuclei.

I would like to turn alow just for a minute to the Coulomb exciters. After ubout six or seven years of starvetion it looks as if very rich years may be coming. It seems they have learned how to handle all the small interference effects, which they now use in measuring quadrupole moments in a model 
independent way and also El-transition probabilities. With these measuremints one probably can obtain very nice data on collective properties of excited states. New prospects also lie ahead with the heavy-ion beams. Lee Grodzins tried very hard to show us how difficult that game may be, but I can promise him that he didn't scare me, as I am now jolning that club which will try to use the heavy-ion beams to do Coulomb excitation and similar experiments. Evca if you consider the very complicated behavior of the excitation probabilities of highly excited states, you still find that it is possible to excite them; and if you excite them then you immediately have a great deal of information about energy, angular momentum and such things for these very highly excited states, which may produce some surprises. In addition, I think the higher recoil veloeities will allow us to do all kinds of new measurements, perhaps even to determine lifetimes of high angular-momentum states directly by use of recoil techniques.

Now I think I an at the end of my talk and Gerry Brown nay think that I missed the physics of the conference and that may well be true; and it may well have been that all I saw and all that I was trying to tell you was a dream. However, there was one thing which I saw and appreciated the most and which was the most important thing at this conference and that $w^{\prime}=$ the great hospitality of our Japanese friends who made a tremendous effort in arranging this meeting in order to bring us closer together; all those who like this field and even those who don't. I should like t., express my deep appreciation and sincere thanks to Professor Sugimoto and his organizing committee for providing us with such a comfortable meeting and a most exciting exhibition of the most artistic field in nuclear physics to which they themselves have 
contributed to such a great extent. Now let me close my talk with the most simple but sincere phrases I could find and say arigato and sayonara!! Auf Wiedersehen und Danke Schön!! 